\title{
Etude du transport de galets par les torrents au moyen de traceurs radioactifs
}

\author{
F. Tola \\ Service d'application des radioéléments, CEA Saclay
}

\section{Introduction}

L'aménagement des cours d'eau, la construction des retenues, l'extraction des matériaux du lit..., nécessitent une bonne connaissance du transport par charriage des rivières alluvionnaires, afin d'en prévoir les conséquences.

Ce transport très complexe fait intervenir un trop grand nombre de paramètres pour pouvoir être traité mathématiquement. Par ailleurs, l'extrapolation des formules semi-empiriques classiques, du type MEYERPeter ou Einstein, calées en canal de laboratoire, ne permet pas de l'estimer avec une marge de sécurité suffisante [1]. Quant aux incertitudes liées à la définition des grandeurs géométriques, qui varient d'une section à l'autre, à l'instationnarité de l'écoulement en période de charriage, à l'étendue de la courbe granulométrique, à la difficulté d'évaluer certains paramètres et facteurs correctifs..., le problème a déjà été soulevé par de nombreux chercheurs [2 à 4 ].

L'emploi de galets marqués par un radioisotope $\gamma$, aisément détectable in situ, permet une étude à la fois qualitative et quantitative du phénomène. Depuis 1968, le service d'applications des radioéléments du C.E.A. Saclay effectue ce genre d'expérience pour le compte d'organismes divers. Citons parmi celles-ci :

- dans les Pyrénées Orientales : sur le Verdouble, le Cady, le Tet et la Lentila, dans le cadre de l'aménagement en eau de la plaine du Roussillon (barrage de Vinça); sur l'Agly (pour le compte de la D.D.E. de l'Agriculture), en vue d'édifier un barrage dans le secteur de Caramany (Corbières Méridionales) ;
- dans les Vosges, sur la Bruche, la Mossig et la Doller (à la demande du génie rural du Haut-Rhin), dans le cadre de l'aménagement des cours d'eau vosgiens et d'un lac collinaire à Pont d'Aspach,

- en Haute Corse, sur le Fium'Orbo (pour le compte d'EDF), en amont de l'actuel barrage de Sampolo,

- dans la Somme, le long du littoral des Bas-Champs, pour la D.D.E. de la Somme.

Un maximum d'informations peuvent être recueillies lorsque les détections s'échelonnent entre les crues. Outre le débit solide, elles portent sur les conditions critiques d'entraînement, la longueur et la vitesse moyenne de déplacement des galets, les chenaux favorisés, les zones préférentielles de dépôt ou d'érosion, ainsi que la largeur et l'épaisseur de la couche en mouvement.

Après un bref rappel de la méthodologie, nous illustrerons les résultats à partir des expériences réalisées sur l'Agly.

\section{Méthodologie}

En raison de sa durée de vie et de son pouvoir de pénétration, il est devenu classique d'utiliser comme radioélément marqueur, le Tantale 182 , de 115 jours de période et d'énergie $\gamma$ comprise entre 0,07 et $1,2 \mathrm{MeV}$. Celui-ci permet de suivre les galets sur un laps de temps de 1 an et leur détection jusqu'à une épaisseur d'enfouissement de $0,80 \mathrm{~m}$. Cette contrainte rend les mesures impossibles en période de grande crue, car alors le remaniement en profondeur est trop important.

The study of the transport of pebbles by torrents by means of radioactive tracers

Conservancy of rivers and, particularly, the construction of dams need an estimation of the transport of pebbles by torrents located up stream. This transport involves a great number of parameters, most of which mathematical models are unable to take into account. Thus, extrapolation of semi-emperical formulaes actually available does not allow precise quantification within a reasonable safety margin. The use of pebbles labelled with a radioisotope easily detectable in situ, tantale 182 (half-life 114 days, energy from 0,07 to 1,2 MeV), allows, in most cases, both a qualitative and quantitative study of solids transport by torrents. Numerous experiments, from 1968 til today have permitted to improve and optimize the method. 
Le marquage se fait en perforant chaque galet d'un trou borgne dans lequel on introduit un fil de Tantale, de $5 \mathrm{~mm}$ de longueur et $0,3 \mathrm{~mm}$ de diamètre. Le trou est ensuite bouché à l'aide d'une résine thermodurcissable. En raison du nombre de galets marqués, environ 500, cette opération peut se dérouler directement sur le site. L'activité par galet, $100 \mu \mathrm{Ci}(3,7 \mathrm{MBq})$ est inférieure à l'activité maximale définie par les conditions particulières d'emploi, soit $140 \mu \mathrm{Ci}(5,18 \mathrm{MBq})$ [5]. La granulométrie des galets marqués est représentative de celle du lit.

L'immersion s'effectue en période d'étiage, en remplaçant les galets du lit par des galets marqués de dimensions semblables.

Les détections ont lieu, selon les conditions hydrauliques, soit à pied sur les bancs découverts, soit en cuissardes par faibles profondeurs, soit en bateau pneumatique dans les trous d'eau profonds, ou encore, lorsque la nature du lit le permet, par va-et-vient d'un traîneau porte-sonde d'une rive à l'autre. On relève systématiquement la position ainsi que l'intensité du rayonnement à l'aplomb de chaque galet repéré.

Les appareils de détection sont des scintillomètres portatifs dont l'élément sensible au rayonnement $\gamma$ est un cristal d'iodure de sodium activé au thallium.

A partir de ces mesures, et grâce à un étalonnage préalable en laboratoire, on détermine la profondeur d'enfouissement de chaque galet, en tenant compte du bruit de fond du lit, dont un relevé systématique est effectué avant l'immersion. Les galets accessibles sont déterrés afin de déterminer leur granulométrie, leur usure, et de vérifier la validité de l'étalonnage précédent.

Le taux de récupération est compris en 30 et $80 \%$, certains galets échappant à la détection, soit parce que certaines zones sont restées inexplorées (présence de rapides, d'étroits défilés ou de trous profonds), soit à cause de l'importance de l'enfouissement et/ou de la décroissance radioactive.

\section{Exemple d'application : Etude du déplacement de galets radioactifs sur l'Agly}

Afin d'évaluer le transport par charriage de l'Agly en amont du futur barrage de Caramany, on a procédé à deux campagnes de mesures avec des traceurs radioactifs. Les résultats complets sont rapportés dans [6]. A cette occasion, on effectua une étude comparative des procédés de marquage radioactif et magnétique, afin de définir les avantages et inconvénients ainsi que les limites d'application de chaque méthode. Malgré une grande facilité de marquage des galets supérieurs à $5 \mathrm{~cm}$, l'absence de risque pour l'opérateur et le milieu naturel, une durée illimitée et une meilleure sensibilité permettant en particulier la détection de galets très enfouis, la méthode magnétique présente de nombreux inconvénients, notamment : la lenteur de la détection, due à un appareillage complexe et peu simple d'emploi qui pourrait être perfectionné dans l'avenir, la gêne due au bruit de fond créé par les nombreux objets métalliques présents dans la nature, le manque de précision sur la position d'un galet, l'impossibilité de distinguer des galets voisins et donc d'en définir le nombre, l'absence de loi permettant de définir la profondeur d'enfouissement.
Une première campagne se déroulant entre septembre 1975 et août 1976, a permis d'estimer à $155 \mathrm{t}$ le charriage au cours de cette période. Celui-ci peut être attribué à une crue unique dont le maximum a atteint $164 \mathrm{~m}^{3} \cdot \mathrm{s}^{-1}$, valeur légèrement supérieure au débit critique d'entrâ̂nement des galets de 7,5 à $9,5 \mathrm{~cm}$. Tous les galets supérieures à $10 \mathrm{~cm}$ sont restés sur la zone d'immersion, tandis que les petits galets de 4 à $5 \mathrm{~cm}$ ont subi un transport superficiel sur une épaisseur de $8 \mathrm{~cm}$, certains ayant été retrouvés jusqu'à $800 \mathrm{~m}$ du point d'immersion. Nous décrivons plus en détail la deuxième campagne ciaprès.

En aval du pont de Caramany, site où s'est déroulé l'expérience, la largeur de l'Agly est d'environ $15 \mathrm{~m}$ et sa pente varie dans une fourchette comprise entre $7 \times$ $10^{-4}$ et $10^{-2}$

La figure 1 donne l'enveloppe granulométrique, ainsi que la courbe granulométrique moyenne du lit. Elle a été obtenue à partir d'une série d'échantillons prélevés en différents points de 7 sections droites, en même temps qu'on procédait à une étude topographique et morphologique des lieux (lever des profils transversaux, du profil longitudinal, description des berges et du lit, recherche de baisses de crue, etc...).

Compte-tenu de cette courbe, 450 galets ont été marqués au Tantale 182 et décomposés en 4 classes granulométriques, dans les proportions suivantes: 200 galets de diamètre compris entre 4 et $5 \mathrm{~cm}(45 \%)$, 100 galets de diamètre compris entre 7,5 et $8,5 \mathrm{~cm}$ $(22,5 \%), 100$ galets de diamètre compris entre 8,5 et $9,5 \mathrm{~cm}(22,5 \%), 50$ galets de diamètre compris entre 10 et $14 \mathrm{~cm}(10 \%)$.

Entre la date d'immersion, en aoüt 1976 et la période de détection, du 17 au 27 juillet 1977, une seule crue importante a eu lieu, son maximum atteignant $216 \mathrm{~m}^{3} \cdot \mathrm{s}^{-1}$ (fig. 2). 359 galets, soit $80 \%$ des galets marqués, ont été retrouvés, répartis sur toute la largeur de la rivière. 215 ont été déterrés $(48 \%)$ afin de déterminer leur granulométrie et de vérifier leur profondeur d'enfouissement. Leur répartition longitudinale est représentée sur la figure 3 , tandis que la figure 4 donne cette même répartition par classes granulométriques, pour les galets déterrés. On peut faire les constatations suivantes :

- 115 galets, soit $32 \%$ du nombre total détecté, dont $15 \%$ qui n'ont pas quitté la zone d'immersion, sont restés dans les 30 premiers mètres. En particulier, tous les gros galets de 10 à $14 \mathrm{~cm}$, s'y trouvent concentrés et ne participent pas au transport ;

$-20 \%$ des petits galets $(4$ à $5 \mathrm{~cm}$ ) sont restés dans les 40 premiers mètres, dû à l'effet combiné du pavage et du piégeage sous de gros blocs;

- on retrouve 70 galets $(20 \%)$ entre 100 et $300 \mathrm{~m}$, qui est la distance limite de transport des galets de 7,5 à $9,5 \mathrm{~cm}$;

- il n'y a pas de différence de comportement appréciable entre les deux classes granulométriques de 7,5 à $8,5 \mathrm{~cm}$ et 8,5 à $9,5 \mathrm{~cm}$;

- 26 galets $(7 \%)$ ont été retrouvés entre 300 et $500 \mathrm{~m}$, zone de dépôt intensif, où la largeur de l'Agly passe par un maximum, pour une pente minimale. Une bonne partie des galets ayant franchi $300 \mathrm{~m}$ se dépose dans cette 


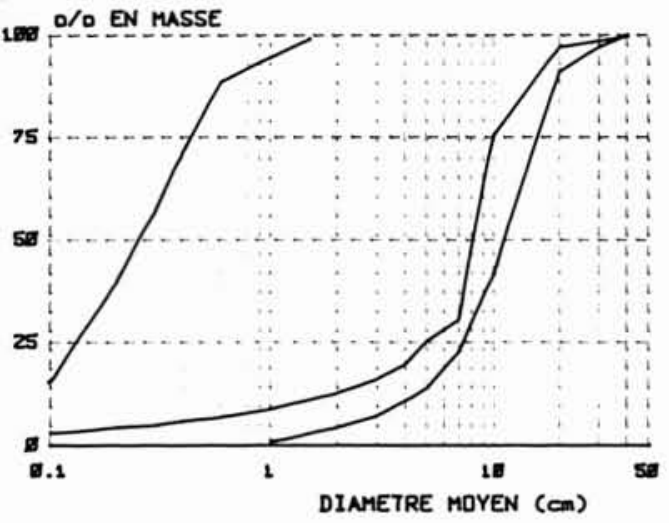

1. Enveloppe et courbe granulométrique moyenne de l'Agly à Caramany.

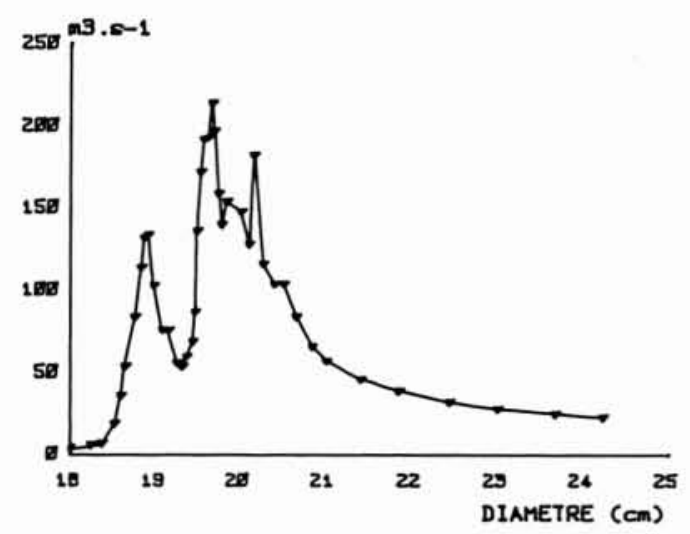

2. Hydrogramme de la crue de mai 1977 à Planèzes.

zone, où ils sont recouverts en fin de crue par une épaisseur notable de matériaux fins. C'est vraisemblablement ici que se trouve bon nombre de galets non détectés, à cause de leur enfouissement ;

- on retrouve 115 galets $(32 \%)$ entre 500 et $800 \mathrm{~m}$, zone de piégeage due à la présence de bancs entre deux bras de la rivière. Il semble très improbable que des galets aient franchi cette zone, qui constitue une barrière naturelle.

Le centre de gravité en nombre de l'ensemble des galets détectés se trouve à $280 \mathrm{~m}$ du point d'immersion. On peut chercher à apporter une correction à cette valeur en admettant un rendement de détection de $90 \%$ entre 0 et $300 \mathrm{~m}, 50 \%$ entre 300 et $500 \mathrm{~m}$ et $75 \%$ entre 500 et $800 \mathrm{~m}$. Ceci nous conduit à rajouter 24 galets dans la première zone, 26 dans le deuxième et 38 dans la dernière. Le centre de gravité de la population marquée se trouverait alors à $308 \mathrm{~m}$ du point d'immersion, soit un écart de $10 \%$ par rapport à la valeur précédente. Nous prendrons :

$$
l_{G}=295 \pm 15 \mathrm{~m} .
$$

La figure 5 donne la répartition en profondeur des galets retrouvés à moins de $300 \mathrm{~m}$ du point d'immersion. Le transport intéresse essentiellement une couche de $20 \mathrm{~cm}$ d'épaisseur, avec un transport superficiel prépondérant ( $80 \%$ sur les 12 premiers $\mathrm{cm})$.

La figure 6 concerne uniquement les galets retrouvés au-delà de $300 \mathrm{~m}$. Ici, la répartition en profondeur est quasi homogène sur une épaisseur de $35 \mathrm{~cm}$, ce qui

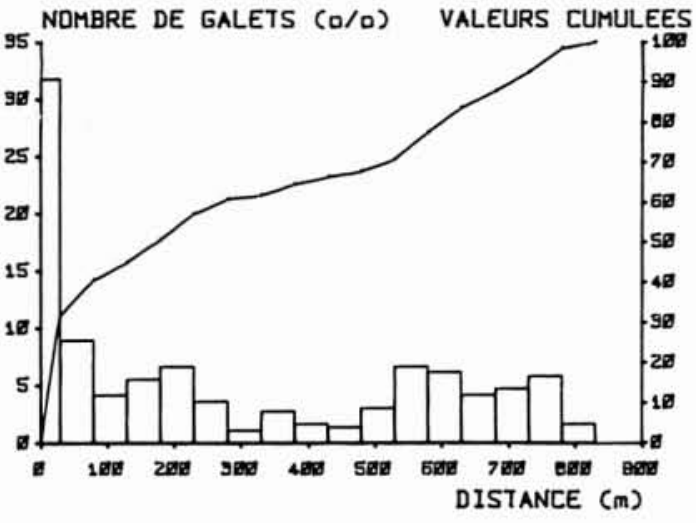

3. Répartition longitudinale des galets.

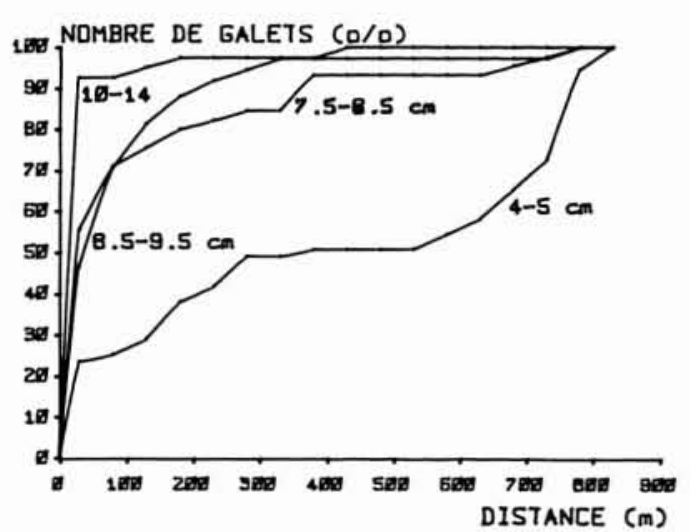

4. Répartition longitudinale de chaque classe granulométrique (galets déterrés).

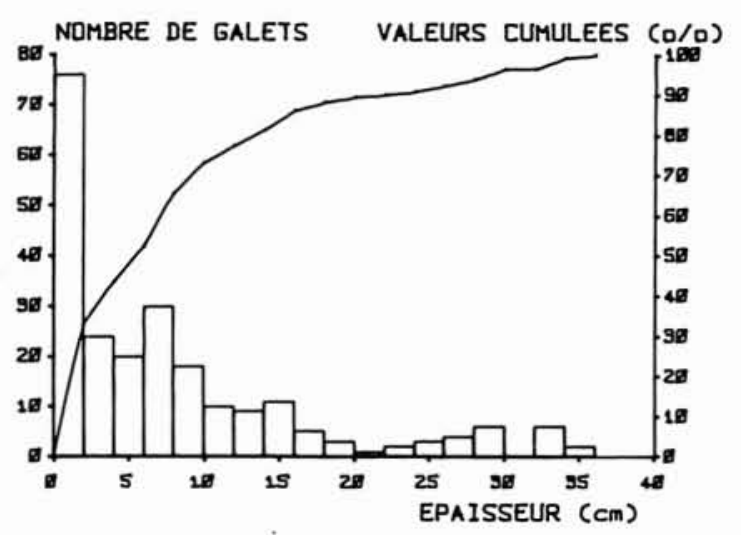

5. Répartition en profondeur des galets retrouvés à moins de $300 \mathrm{~m}$ de la zone d'immersion.

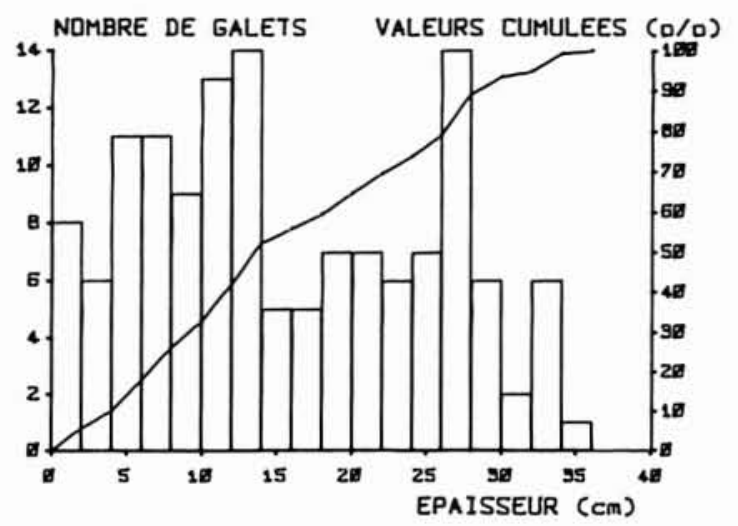

6. Répartition en profondeur des galets retrouvés au-delà de $300 \mathrm{~m}$. 
dénote un remaniement important. Il serait cependant inexact de prendre cette valeur comme épaisseur de transport. En effet, comme nous l'avons souligné précédemment, la zone comprise entre 300 et $500 \mathrm{~m}$ est une zone de recouvrement où le transport s'effectue sur une épaisseur de 20 à $25 \mathrm{~cm}$ seulement, tandis que, au-delà de $500 \mathrm{~m}$, il y a atterrissement sur les bancs locaux sur environ $40 \mathrm{~cm}$ d'épaisseur. En conséquence, on peut estimer que l'épaisseur moyenne de transport vaut :

$$
E=25 \pm 5 \mathrm{~cm} \text {. }
$$

Si on admet que la répartition en nombre des galets marqués dans chaque classe est représentative de la répartition massique des sédiments du lit, alors, le volume charrié par l'Agly au cours de la période d'étude aura été :

$$
\begin{aligned}
G_{s} & =l_{G} \times E \times L=(295 \pm 15) \times(0,25+0,05) \times 15 \\
& =1115 \pm 275 \mathrm{~m}^{3},
\end{aligned}
$$

où $l_{G}=\frac{1}{N} \sum_{i=1}^{N} l_{i}$, est le centre de gravité en nombre des galets marqués, $E$, l'épaisseur moyenne de transport, et, $L$, la largeur moyenne de transport.

Il s'agit essentiellement de galets inférieurs à $10 \mathrm{~cm}$, les plus gros galets ne participant pas au transport.

Il est également possible de déterminer les quantités charriées pour chaque classe granulométrique, à condition d'en marquer et déterrer un nombre significatif par classe. $l_{G i}$ désignant le centre de gravité de la $i$ ième classe et $P_{m i}$ sa fraction massique en tant que constituant du lit (mais est-il possible de la définir de manière précise?), on a :

$$
G_{s i}=P_{m i} \times l_{G i} \times E \times L,
$$

expression qui dans le cas présent conduit aux valeurs

\begin{tabular}{|c|c|c|c|c|c|}
\hline Granulométrie $(\mathrm{cm})$ & $P_{m}$ & $I_{G}(\mathrm{~m})$ & $E(\mathrm{~cm})$ & $L(m)$ & $G_{s}\left(m^{3}\right)$ \\
\hline $4-5$ & $50 / 450$ & 476 & \multirow{3}{*}{25} & \multirow{3}{*}{15} & 793 \\
\hline $7,5-9,5$ & $200 / 450$ & 214 & & & 356 \\
\hline $10-14$ & $200 / 450$ & 15 & & & 6 \\
\hline
\end{tabular}
indiquées dans le tableau ci-dessous:

\section{Grandeurs caractéristiques du transport pour chaque classe granulométrique}

Le volume total ainsi obtenu, $1155 \mathrm{~m}^{3}$, est à comparer à celui que nous avions trouvé plus haut par des considérations globales. Evidemment, on pourrait reprendre ce calcul en adoptant une répartition massique que l'on jugerait plus proche de la répartition réelle.

Remarquons enfin que, compte-tenu des difficultés et des incertitudes énumérées dans notre introduction, toute tentative de calcul à partir des formules empiriques devient vite un exercice de haute voltige et le résultat illusoire. En particulier, les formules du type MEYERPETER conduisent à l'absence de transport, la contrainte tangentielle sur le lit étant toujours inférieure à la contrainte critique d'entraînement des matériaux. Ceci illustre bien le manque de précision de telles formules au voisinage des conditions critiques.

\section{Conclusion}

Bien que la méthode des traceurs ait évolué, en se perfectionnant au fil des années, certaines contraintes n'ont pu être surmontées. Citons parmi celles-ci, l'impossibilité de détecter des galets enfouis au-delà de $80 \mathrm{~cm}$. Par ailleurs, des questions relatives à la représentativité et la précision de la méthode restent encore posées :

Quel critère doit-on utiliser pour répartir les galets par classe ? Car, peut-on définir une courbe granulométrique unique pour les matériaux du lit ? Et, si oui, comment?

Peut-on justifier l'emploi du déplacement moyen des galets dans le calcul du charriage ?

Avec quelle précision le centre de gravité des galets retrouvés représente-t-il celui de la population?

Nous ne sommes pas capables de répondre concrètement à ces questions. Cependant, l'expérience montre que la méthode des traceurs fournit une bonne estimation des quantités charriées, avec, dans le pire des cas, une incertitude de 50 à $100 \%$, ce qui est peu de chose lorsqu'on sait qu'il y a facilement un facteur 10 d'écart entre les diverses formules empiriques.

Finalement, nous sommes convaincus qu'une étroite collaboration entre hydrauliciens, sédimentologues et spécialistes de la radioactivité permettrait de faire avancer nos connaissances dans le domaine du transport de sédiments, le problème étant suffisamment complexe pour qu'on ne néglige aucun moyen de l'aborder. Par son coût modéré (environ 150000 francs pour une expérience d'une durée de un an) et la richesse des informations fournies, la méthode radioactive devrait, en tant que seul moyen de vérification et de calage en nature, trouver sa place à côté des modèles physiques et numériques.

\section{Références}

[1] M. Bouvard. - Ouvrages de dérivation et transports solides. La Houille Blanche. N" spécial 3-4/1983.

[2] G. Courtors et al.. - L'application des radioéléments en sédimentologie. La Houille Blanche, N* 7/1970, Annexes 3.1 et 3.2 .

[3] C. CERTES. - Etude de déplacements de galets radioactifs sur le Fium'Orbo: Comparaison des résultats expérimentaux obtenus et de l'application de la formule de Meyer-Peter. Rapport SAR/ORIS 83 15, 15 juin 1983.

[4] P. Y. FAfournoux. - Etude bibliographique et théorique de la capacité de transport par les fleuves et les rivières. Rapport Général. Laboratoire Central d'Hydraulique de France, septembre 1971.

[5] G. Courtols, R. Hours. - Propositions concernant les conditions particulières d'emploi des radioéléments artificiels pour étudier les mouvements de sédiments. Rapport SAR, 1964.

[6] G. Courtois et al..-Etude comparative des procédés radioactifs et magnétiques concernant les déplacements de galets. Rapport DCA/SARR C 250, février 1978. 